\title{
Early Experiments with a New In Situ SEM Heating Technology
}

\author{
Jane Y. Howe,* Larry R. Walker,* Lawrence F. Allard,* and Hendrix Demers** \\ * Materials Science and Technology Div., Oak Ridge National Laboratory, Oak Ridge, TN 37831 \\ ** Département de Génie Electrique et de Génie Informatique, Université de Sherbrooke, \\ Sherbrooke, Québec Canada J1K 2R1
}

A new heating technology (Protochips, Inc.) has recently been introduced for in situ electron microscopy studies, including both TEM and SEM applications. Aduro ${ }^{\mathrm{TM}}$ heater devices, fabricated using MEMS-based processing [1], offer numerous advantages for in situ SEM applications over those using a miniaturized furnace-type heating cell. The device supports a sample on a thin ceramic membrane that is directly heated by passing a current of a few $\mathrm{mA}$, thereby also heating the sample. The entire heated area of the device is only 500um x 500um, so it is operated without circulating cooling water. The extremely small heated zone enables a rapid temperature response $\left(>10^{4}{ }^{\circ} \mathrm{C} / \mathrm{s}\right)$ for both heating and cooling, and it emits a far lower signal of photons and thermal electrons than a typical furnace-type heating device [2]. This allows heating to temperatures in excess of $1000^{\circ} \mathrm{C}$ without totally degrading the secondary electron signal. The purpose of the present work is to characterize the factors that influence the image quality and resolution of the SEM during heating. We are interested in understanding relative changes of signal-to-noise levels and contrast in secondary electron (SE) and backscattered electron (BSE) images as a function of increasing the heating temperature. We have also assessed the capability for performing energy dispersive X-ray spectroscopy (EDS) during heating experiments.

The in situ holder and the structure of a standard heater chip used in this study are shown in Fig. 1. The chip has an array of $5 \mu \mathrm{m}$ holes in the electrically conductive heater membrane, separated by electrode "fingers" in this case. A C-flat ${ }^{\mathrm{TM}}$ holey carbon film is overlaid on top of the heater membrane. The heating experiments were conducted in a Hitachi S3400 VP-SEM, operated at $15 \mathrm{kV}$ and equipped with an EDAX Si(Li) detector. Fig. 2 shows an Ir-doped FeSi particle situated near the edge of the hole in the membrane, imaged with secondary electrons through an Everhart-Thornley (ET) detector in the specimen chamber, at (2a) RT and at $(2 \mathrm{~b}) 1200^{\circ} \mathrm{C}$, respectively. The image contrast of the low- $Z$ region of the particle was characterized by an intensity profile across the edge of the holey carbon film against the hole (vacuum). Indicated by Line \#1, the contrast difference was $48 \%$ at RT, which dropped to $22 \%$ at $1200^{\circ} \mathrm{C}$. However, the contrast difference of the medium$\mathrm{Z}$ particle against the low-Z support was well above $30 \%$ at $1200^{\circ} \mathrm{C}$ (Line \#2). Heating had a more adverse effect on the contrast of BSE micrographs (not shown), likely caused by the direct detection of photons by solid-state BSE detector. The upper limit for acquiring a BSE micrograph of the low$\mathrm{Z}$ material was about $850^{\circ} \mathrm{C}$ as the contrast was completely washed out by $900{ }^{\circ} \mathrm{C}$. EDS analyses carried out during heating showed effects primarily for the collection of low-energy X-rays $(<1.2 \mathrm{keV})$ below a temperature of $600{ }^{\circ} \mathrm{C}$ : the background counts increased with temperature, thus reduced the signal-to-noise ratio. The upper limit for EDS analysis during heating was about $700^{\circ} \mathrm{C}$.

The decrease in the signal-to-noise ratio for electron detection and EDS spectrum acquisition is mainly due to the emission of thermal electrons and photons at elevated temperatures. The emitted photons are not position sensitive, thus they uniformly raise the background level of a grayscale BSE image (photons do not affect the signal of the ET detector). However, thermal electrons in the range 
of $\sim 0.1 \mathrm{eV}$ are collected by the ET detector, and thereby reduce contrast in the image. It is impressive that even at the highest temperature achievable by the Aduro device $\left(1200^{\circ} \mathrm{C}\right)$, acceptable contrast in SE images is achieved. This is because of the extremely small emitting area of the heater membrane. One solution for improving the contrast of an SE micrograph is to suppress the thermal electrons by a small local positive bias. There are two approaches: 1) manipulating the positive bias of the ET detector or 2) using an energy filter to reduce or remove the thermal electrons. A combined Monte Carlo simulation and experimental study is underway to determine the optimal parameters for minimal detection of thermal electrons. We expect the findings from this study will allow for the optimization of the image contrast and EDS analysis[3].

\section{References}

[1] L. F. Allard, et al., Micros. Res. \& Tech., 72(3) 208-215 (2009).

[2] G. Gregori, H-J. Kleebe, F. Siegelin, G. Ziegler, J. Elect. Micros. 51(6): 347-352 (2002).

[3] Microscopy research at the Oak Ridge National Laboratory's High Temperature Materials Laboratory was sponsored by the U. S. Department of Energy, Office of Energy Efficiency and Renewable Energy, Vehicle Technologies Program.
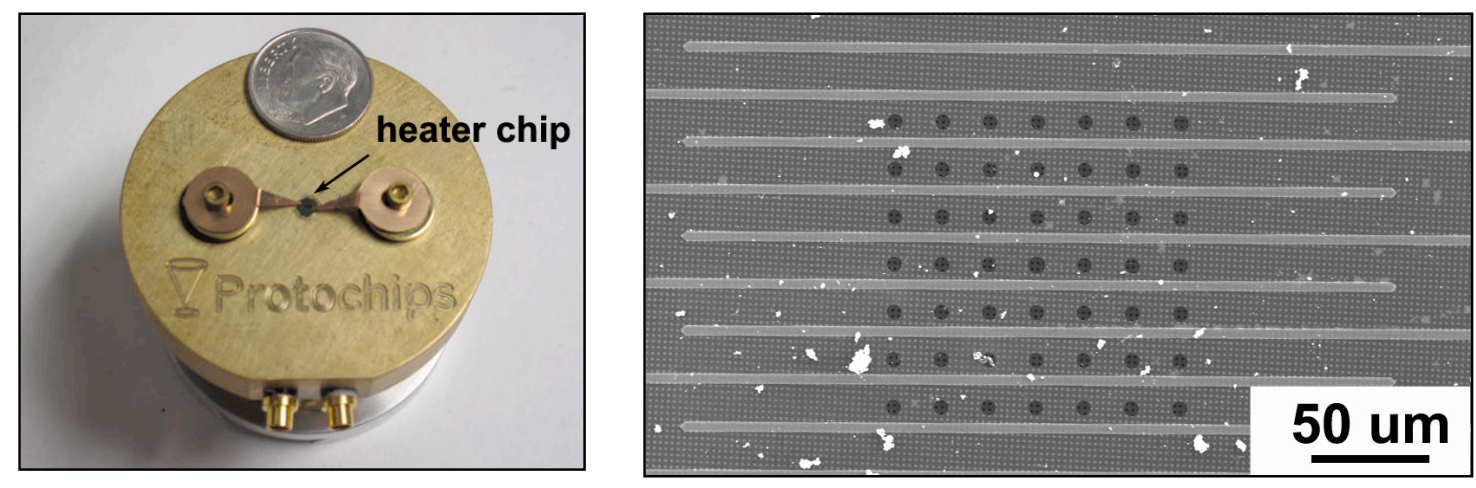

FIG. 1. (A) The heating device (a dime is shown for size comparison), and (B) the enlarged area of the heater membrane of an Aduro ${ }^{\mathrm{TM}}$ chip, showing electrodes.
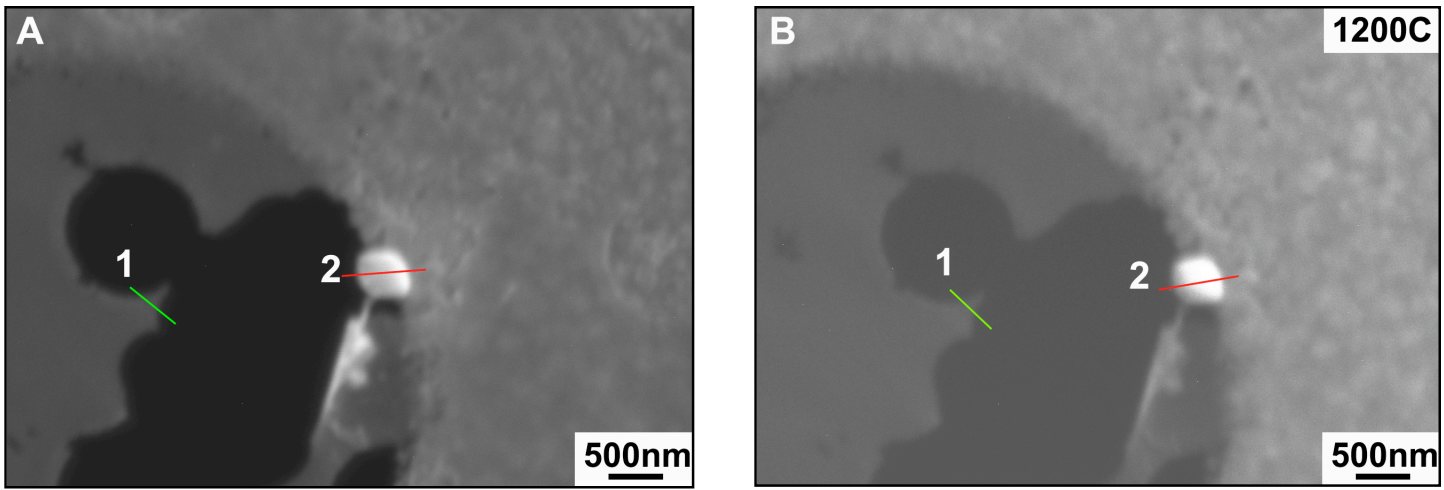

FIG. 2. Iridium-doped FeSi particle situated near the edge of a holey carbon film and the ceramic membrane at (A) RT and (B) $1200^{\circ} \mathrm{C}$. 